\title{
Health Care Disparities of Ohioans With Developmental Disabilities Across the Lifespan
}

\author{
Jessica A. Prokup, RN, BSN ${ }^{1,2}$ \\ Rebecca Andridge, $\mathrm{PbD}^{3}$ \\ Susan M. Havercamp, $\mathrm{PbD}^{4}$ \\ Emily A. Yang, MPH
}

'Medical Student Research Program, The Ohio State University College of Medicine, Columbus, Ohio

${ }^{2}$ The Ohio State University, Nisonger Center, Leadership Education in Neurodevelopmental Disabilities (LEND),

Columbus, Ohio

${ }^{3}$ Division of Biostatistics, The Ohio State University College of Public Health, Columbus, Ohio

${ }^{4}$ The Ohio State University, Nisonger Center, Ohio Disability \& Health Program, Columbus, Ohio

Conflicts of interest: authors report none.

\section{CORRESPONDING AUTHOR}

Susan Havercamp, PhD OSU Nisonger Center 371G McCampbell Hall 1581 Dodd Dr

Columbus, OH 43210

susan.havercamp@osumc.edu

\begin{abstract}
We explored health care differences across the lifespan comparing people with developmental disabilities to people without developmental disabilities. Health care disparities are inequities occurring during the provision of and in access to health care that are experienced by socially disadvantaged populations. We discovered significant disparities between persons with and without developmental disabilities in health status, quality, utilization, access, and unmet health care needs. Our results highlight the need to educate health care clinicians on the care of patients with developmental disabilities of all ages.

Ann Fam Med 2017;15:471-474. https://doi.org/10.1370/afm.2108.

\section{INTRODUCTION}

$\mathrm{D}$ evelopmental disability training is lacking in medicine and public health even though this population has major health needs and poor health outcomes. Developmental disabilities are severe, chronic conditions that impair cognitive and/or physical functioning, are manifest before the age of 22 years, result in functional limitations in at least 3 major activities of daily living, and require assistance throughout the lifespan. ${ }^{1}$ Transitioning from pediatric to adult care can be challenging for young adults with developmental disabilities because of their increased health and support needs. ${ }^{2-4}$ Unfortunately, the health needs of adults with developmental disabilities are rarely addressed in medical training. Using Ohio Medicaid Assessment Survey (OMAS) data, we explored differences on a range of health indicators between populations of those who have developmental disabilities and those do not across their lifespans.
\end{abstract}

\section{METHODS}

We conducted a retrospective cross-sectional analysis of the 2015 OMAS This stratified, random digit-dialing telephone survey is designed to collect health and health care data from Ohio's Medicaid and non-Medicaid populations. In 2015, researchers completed 42,876 interviews with adults and 10,122 proxy interviews for children aged 0 to 18 years (completed by the adult most knowledgeable about the child's health). The OMAS uses single imputation to handle missing data; all indicators used had fewer than $10 \%$ missing responses. Details on the survey design are available elsewhere. $^{5}$

We constructed 2 mutually exclusive groups, (those who had developmental disabilities and those who did not), which were further subdivided by age: children ( 0 to 18 years), those in health care transition (19 to 30 years), adults (31 to 64 years), and older adults (65 years and older). A successful health care transition requires finding appropriate adult medical and specialty health care. This effort is challenging for young adults with disabilities, as many clinicians who treat older adults are uncomfortable caring for this population. Consequently, many young adults with disabilities continue to see pediatric clinicians well into adulthood. ${ }^{6}$ Consistent with previ- 
ous studies, ${ }^{7}$ we defined health care transition as ranging from 19 to 30 years to fully capture young adults with developmental disabilities in transition.

The group comprised children whose adult proxy replied yes to each of the following questions: (1) Does [child] currently have a developmental disability? (2) Is [child] limited or prevented in any way in [his/her] ability to do things most children of the same age can do? (3) Is this because of any medical, behavioral or other health condition? (4) Is this a condition that has lasted or is expected to last for at least 12 months? A response of no to any of these questions resulted in the child being assigned to the no disability group. ${ }^{9}$

The adult survey questionnaire was administered to individuals aged 19 years and older and was completed by a proxy, if necessary. Adults were assigned to the developmental disability group if they endorsed having developmental disabilities and reported at least 1 special health care need, including needing assistance with activities of daily living or receiving special therapies, treatment, or counseling. ${ }^{8}$ The special health care need requirement was intended to capture those persons meeting criteria for the federal definition of developmental disabilities. Respondents were classified to the no disability group if they denied having developmental disabilities or denied having any special health care needs.

\section{Statistical Analyses}

We conducted bivariate comparisons between the 2 groups on selected health indicators. We used weights provided in
Table 1. Health Indicators of Respondents

\begin{tabular}{|c|c|c|c|}
\hline Indicator & $\begin{array}{c}\text { Developmental } \\
\text { Disability } \\
\%(95 \% \mathrm{Cl})\end{array}$ & $\begin{array}{c}\text { No Developmental } \\
\text { Disability } \\
\%(95 \% \mathrm{Cl})\end{array}$ & $\begin{array}{l}\text { Prevalence } \\
\text { Ratio } \\
\%(95 \% \mathrm{Cl})^{\mathrm{a}}\end{array}$ \\
\hline \multicolumn{4}{|c|}{ Health status: fair or poor } \\
\hline $0-18 y^{b}$ & $27.7(22.7-32.6)$ & $1.1(0.8-1.4)$ & $25.3(18.5-34.6)^{c}$ \\
\hline $19-30 y^{d}$ & $42.8(33.0-52.6)$ & $6.7(5.9-7.6)$ & $6.3(4.9-8.2)^{c}$ \\
\hline $31-64 y^{e}$ & $71.0(66.6-75.3)$ & $11.3(10.7-11.8)$ & $6.3(5.8-6.8)^{c}$ \\
\hline$\geq 65 y^{f}$ & $76.0(67.4-84.6)$ & $4.3(3.8-4.9)$ & $4.3(3.8-4.9)^{c}$ \\
\hline \multicolumn{4}{|l|}{ Utilization $^{g}$} \\
\hline \multicolumn{4}{|c|}{$\geq 2$ Overnight hospitalizations } \\
\hline $0-18$ y & $8.5(5.5-11.4)$ & $0.7(0.5-.0 .9)$ & $12.3(7.7-19.6)^{c}$ \\
\hline $19-30$ y & $14.2(7.0-21.5)$ & $2.3(1.8-2.8)$ & $6.1(3.5-10.7)^{c}$ \\
\hline $31-64$ y & $13.8(10.8-16.8)$ & $2.2(2.0-2.5)$ & $6.2(4.8-7.9)^{c}$ \\
\hline$\geq 65 y$ & $17.5(10.2-24.8)$ & $4.6(4.0-5.1)$ & $3.8(2.5-5.9)^{c}$ \\
\hline \multicolumn{4}{|c|}{$\geq 2$ Emergency department visits } \\
\hline $0-18$ y & $23.8(19.1-28.6)$ & $7.0(6.3-7.7)$ & $3.4(2.7-4.3)^{c}$ \\
\hline $19-30 y$ & $37.0(27.4-46.6)$ & $11.6(10.5-12.7)$ & $3.2(2.4-4.2)^{c}$ \\
\hline $31-64$ y & $36.8(32.2-41.4)$ & $6.3(5.9-6.8)$ & $5.8(5.0-6.7)^{c}$ \\
\hline$\geq 65 y$ & $34.5(24.1-44.9)$ & $5.7(5.0-6.3)$ & $6.1(4.4-8.4)^{c}$ \\
\hline \multicolumn{4}{|l|}{ Quality ${ }^{g}$} \\
\hline \multicolumn{4}{|c|}{$\begin{array}{l}\text { Primary care physician } \\
\text { usually or always spends } \\
\text { enough time with patient }\end{array}$} \\
\hline $0-18$ y & $91.6(88.0-95.1)$ & $90.8(89.9-91.8)$ & $1.01(0.97-1.05)$ \\
\hline $19-30 y$ & $70.0(54.5-85.5)$ & $82.6(80.5-84.7)$ & $0.85(0.68-1.06)$ \\
\hline $31-64$ y & $83.2(78.9-87.5)$ & $90.8(90.2-91.5)$ & $0.92(0.87-0.96)$ \\
\hline$\geq 65$ y & $75.5(63.9-87.0)$ & $92.7(91.9-93.5)$ & $0.81(0.70-0.95)^{c}$ \\
\hline \multicolumn{4}{|c|}{$\begin{array}{l}\text { Primary care physician } \\
\text { usually or always explains } \\
\text { things well }\end{array}$} \\
\hline $0-18 y$ & $94.7(91.4-98.0)$ & $96.4(95.8-96.9)$ & $0.98(0.95-1.02)$ \\
\hline $19-30 y$ & $89.7(80.7-98.6)$ & $94.5(93.2-95.7)$ & $0.95(0.86-1.05)$ \\
\hline $31-64$ y & $84.5(80.0-89.0)$ & $96.0(95.5-96.4)$ & $0.88(0.84-0.93)^{c}$ \\
\hline$\geq 65$ y & $83.0(73.2-92.9)$ & $95.0(94.4-95.7)$ & $0.87(0.78-0.98)^{c}$ \\
\hline \multicolumn{4}{|l|}{ Access $^{g}$} \\
\hline \multicolumn{4}{|c|}{ Usual source of care } \\
\hline $0-18$ y & $98.8(97.5-100.0)$ & $95.5(95.0-96.0)$ & $1.03(1.02-1.05)^{\mathrm{h}}$ \\
\hline $19-30 y$ & $88.5(83.0-93.9)$ & $83.2(82.0-84.4)$ & $1.06(1.00-1.13)$ \\
\hline $31-64$ y & $93.0(90.2-95.9)$ & $91.4(90.9-91.9)$ & $1.02(0.99-1.05)$ \\
\hline$\geq 65$ y & 96.7 (93.2-100.0) & $96.1(95.6-96.6)$ & $1.01(0.97-1.04)$ \\
\hline \multicolumn{4}{|c|}{ Delayed treatment } \\
\hline $0-18$ y & $10.1(6.8-13.4)$ & $2.4(2.0-2.8)$ & $4.2(2.9-6.0)^{c}$ \\
\hline $19-30 y$ & $23.2(15.1-31.3)$ & $14.3(13.1-15.5)$ & $1.6(1.1-2.3)^{\mathrm{h}}$ \\
\hline $31-64$ y & $32.5(27.9-37.1)$ & $15.6(15.0-16.3)$ & $2.1(1.8-2.4)^{c}$ \\
\hline$\geq 65$ y & $14.9(7.6-22.2)$ & $6.9(6.2-7.6)$ & $2.2(1.3-3.6)^{h}$ \\
\hline \multicolumn{4}{|c|}{ Problem getting needed care } \\
\hline $0-18$ y & $13.7(10.1-17.4)$ & $2.1(1.7-2.5)$ & $6.4(4.7-8.9)^{c}$ \\
\hline $19-30 y$ & $16.2(9.0-23.4)$ & $5.4(4.6-6.2)$ & $3.0(1.9-4.8)^{c}$ \\
\hline $31-64$ y & $23.1(19.1-27.1)$ & $4.9(4.5-5.3)$ & $4.7(3.9-5.8)^{c}$ \\
\hline$\geq 65$ y & $17.5(8.5-26.5)$ & $2.4(2.0-2.8)$ & $7.3(4.2-12.6)^{c}$ \\
\hline \multicolumn{4}{|c|}{$\begin{array}{l}\text { Received same-day care } \\
\text { urgent needs }\end{array}$} \\
\hline $0-18$ y & $47.5(41.3-53.7)$ & $61.1(59.6-62.7)$ & $0.78(0.68-0.89)^{c}$ \\
\hline $19-30 y$ & $34.7(14.5-55.0)$ & $38.6(34.2-43.0)$ & $0.90(0.50-1.63)$ \\
\hline $31-64$ y & $28.9(22.3-35.4)$ & $42.8(40.9-44.6)$ & $0.68(0.54-0.85)^{\mathrm{h}}$ \\
\hline$\geq 65$ y & $12.5(1.1-24.0)$ & $42.2(39.5-44.9)$ & $0.30(0.12-0.74)^{\mathrm{h}}$ \\
\hline
\end{tabular}


Table 1. Health Indicators of Respondents (continued)

\begin{tabular}{|c|c|c|c|}
\hline Indicator & $\begin{array}{c}\text { Developmental } \\
\text { Disability } \\
\%(95 \% \mathrm{Cl})\end{array}$ & $\begin{array}{c}\text { No Developmental } \\
\text { Disability } \\
\%(95 \% \mathrm{Cl})\end{array}$ & $\begin{array}{l}\text { Prevalence } \\
\text { Ratio } \\
\%(95 \% \mathrm{Cl})^{\mathrm{a}}\end{array}$ \\
\hline \multicolumn{4}{|c|}{ Waited 2 or more days for urgent needs } \\
\hline $0-18$ y & $29.9(24.3-35.5)$ & $16.1(14.9-17.3)$ & $1.9(1.5-2.3)^{c}$ \\
\hline $19-30$ y & $38.2(17.7-58.6)$ & $34.3(30.0-38.6)$ & $1.1(0.64-1.9)$ \\
\hline $31-64$ y & $48.0(40.8-55.3)$ & $30.2(28.5-31.9)$ & $1.6(1.4-1.9)^{c}$ \\
\hline$\geq 65$ y & $44.0(24.6-63.4)$ & $29.0(26.4-31.5)$ & $1.5(0.97-2.4)$ \\
\hline \multicolumn{4}{|c|}{ Usually or always received needed after-hours care from primary care physician } \\
\hline $0-18$ y & $59.4(53.0-65.7)$ & $61.6(60.0-63.2)$ & $0.96(0.86-1.08)$ \\
\hline $19-30$ y & $53.8(24.0-83.6)$ & $59.6(53.7-65.5)$ & $0.90(0.51-1.59)$ \\
\hline $31-64$ y & $44.9(35.6-54.2)$ & $48.5(45.6-51.4)$ & $0.92(0.75-1.15)$ \\
\hline$\geq 65$ y & $46.8(23.9-69.8)$ & $56.5(51.8-61.2)$ & $0.83(0.50-1.36)$ \\
\hline \multicolumn{4}{|c|}{ Problem seeing a specialisti } \\
\hline $0-18$ y & $24.6(19.2-30.0)$ & $19.3(17.0-21.6)$ & $1.3(0.99-1.6)$ \\
\hline $19-30 y$ & $52.3(38.6-66.1)$ & $26.8(23.7-30.0)$ & $2.0(1.5-2.6)^{c}$ \\
\hline $31-64$ y & $47.6(41.8-53.4)$ & $19.7(18.4-21.0)$ & $2.4(2.1-2.8)^{c}$ \\
\hline$\geq 65 y$ & $45.8(32.5-59.1)$ & $12.9(11.5-14.2)$ & $3.6(2.6-4.9)^{c}$ \\
\hline \multicolumn{4}{|c|}{ Unmet health care need ${ }^{g}$} \\
\hline \multicolumn{4}{|l|}{ Dental } \\
\hline $0-18$ y & $8.3(5.1-11.4)$ & $3.3(2.8-3.8)$ & $2.5(1.7-3.8)^{c}$ \\
\hline $19-30 y$ & $34.2(24.7-43.8)$ & $14.0(12.8-15.2)$ & $2.4(1.8-3.3)^{c}$ \\
\hline $31-64$ y & $34.4(29.9-38.9)$ & $10.0(9.5-10.6)$ & $3.4(3.0-3.9)^{c}$ \\
\hline$\geq 65 y$ & $20.8(12.2-29.3)$ & $5.0(4.5-5.6)$ & $4.1(2.7-6.3)^{c}$ \\
\hline \multicolumn{4}{|l|}{ Vision } \\
\hline $0-18$ y & $5.0(2.6-7.3)$ & $1.8(1.4-2.2)$ & $2.8(1.7-4.6)^{c}$ \\
\hline $19-30 y$ & $28.7(19.4-38.0)$ & $8.0(7.0-8.9)$ & $3.6(2.6-5.1)^{c}$ \\
\hline $31-64$ y & $31.3(26.9-35.7)$ & $9.5(9.0-10.0)$ & $3.3(2.8-3.8)^{c}$ \\
\hline$\geq 65 y$ & $24.4(14.9-34.0)$ & $5.5(4.9-6.1)$ & $4.4(2.9-6.6)^{c}$ \\
\hline \multicolumn{4}{|l|}{ Prescription } \\
\hline $0-18$ y & $9.7(6.4-13.0)$ & $1.7(1.4-2.0)$ & $5.7(3.9-8.4)^{c}$ \\
\hline $19-30 y$ & $24.8(16.2-33.4)$ & $12.4(11.2-13.5)$ & $2.0(1.4-2.9)^{\mathrm{h}}$ \\
\hline $31-64$ y & $28.3(24.1-32.5)$ & $14.2(13.5-14.8)$ & $2.0(1.7-2.3)^{c}$ \\
\hline$\geq 65 y$ & $24.6(15.1-34.0)$ & $9.3(8.5-10.1)$ & $2.6(1.8-3.9)^{c}$ \\
\hline \multicolumn{4}{|c|}{ Other (eg, medical examination, medical supply) } \\
\hline $0-18$ y & $10.6(7.3-13.9)$ & $1.7(1.4-2.0)$ & $6.3(4.4-9.1)^{c}$ \\
\hline $19-30 y$ & $27.5(18.6-36.3)$ & $6.3(5.5-7.2)$ & $4.3(3.1-6.2)^{c}$ \\
\hline $31-64$ y & $26.2(22.0-30.5)$ & $6.1(5.6-6.5)$ & $4.3(3.6-5.2)^{c}$ \\
\hline$\geq 65$ y & $10.5(5.2-15.7)$ & $2.3(1.8-2.7)$ & $4.6(2.7-7.9)^{c}$ \\
\hline \multicolumn{4}{|c|}{$\geq 1$ Unmet need } \\
\hline $0-18$ y & $19.6(15.2-24.0)$ & $5.7(5.0-6.3)$ & $3.5(2.7-4.4)^{c}$ \\
\hline $19-30 y$ & $55.9(46.1-65.8)$ & $25.6(24.1-27.1)$ & $2.2(1.8-2.6)^{c}$ \\
\hline $31-64$ y & $61.2(56.6-65.8)$ & $25.4(24.6-26.1)$ & $2.4(2.2-2.6)^{c}$ \\
\hline$\geq 65$ y & $50.1(39.7-60.3)$ & $16.8(15.8-17.8)$ & $3.0(2.4-3.7)^{c}$ \\
\hline
\end{tabular}

Note: Because individuals with developmental disabilities represent a small percentage of the population, some outcomes were endorsed by a small number of respondents.

a Prevalence ratios and $P$ values are for the difference in health indicators between people with and without developmental disabilities within each age-group.

${ }^{b}$ Developmental disability, $n=419$; no developmental disability, $n=7,701$.

c $p<.001$.

Developmental disability, $n=148$; no developmental disablility, $n=5,225$.

Developmental disability, $n=779$; no developmental disability, $n=19,369$.

f Developmental disability, $n=178$; no developmental disability, $n=10,044$.

${ }^{g}$ During the past 12 months.

h $P \leq .01$.

Among respondents who needed specialist care (19.3\% of children with a developmental disability and $78.4 \%$ of children with no developmental disability did not need specialist care; $34.7 \%$ of adults with a developmental disability aged 19 years and older and $67.1 \%$ of adults with no developmental disability aged 19 years and older did not need specialist care). the OMAS public release data to produce population-level estimates. The Rao-Scott $\chi^{2}$ test was used to test for associations. Because the adult and child survey questions were similar, we were able to compare health indicators across the different age-groups. All analyses were conducted in SAS version 9.4 (SAS Institute Inc).

\section{RESULTS}

Demographic information by age-group and disability status can be found in Supplemental Table 1 at http://www.annfammed.org/content/15/5/471/ suppl/DC1. Study findings are reported in Table $1_{i}$ key findings are summarized here. Adults with developmental disabilities were less likely to have a primary care clinician that spends enough time with them compared with a peer group without developmental disabilities of the same age. Among those with developmental disabilities, older adults were the least likely to have clinicians who explained things well (83.0\%). People with developmental disabilities in all 4 age-groups experienced access barriers, particularly having problems getting needed care and delayed treatment. Finally, children and older adults with developmental disabilities experienced the greatest disparities in unmet health care needs.

\section{DISCUSSION}

We found significant health care disparities for those with developmental disabilities in all 4 age-groups. Although previous research highlighted the difficulty in transitioning 
from pediatric to adult care for young adults with disabilities, ${ }^{6}$ we did not find the young adult age-group (19 to 30 years) to have unique health care disparities. These findings highlight the importance of disability competency for health care clinicians. Health equity in care quality and unmet health care needs may be achieved by incorporating disability education into the undergraduate medical curriculum. Unfortunately, $81 \%$ of medical students reported having had no clinical training in disabilities, ${ }^{9}$ and $58 \%$ of medical school deans reported that a curriculum for patients with disabilities was not a high priority. ${ }^{10}$ Physicians in practice and in training have shown inadequacies in their knowledge of disabilities. ${ }^{10}$

This study is limited by its cross-sectional design, the requirement of spoken English or Spanish, and Ohio residency. Despite careful weighting, response bias and inconsistencies related to self-reported data may have occurred.

With advances in medicine, persons with developmental disabilities are living much longer, medical education, however, has not changed accordingly. To reduce the health care disparities experienced by adults and children with disabilities, medical schools should incorporate training specific to developmental disabilities into their curricula.

To read or post commentaries in response to this article, see it online at http://www.AnnFamMed.org/content/15/5/471.

Key words: disability; developmental disability; developmental delay; intellectual disability; special needs; transition; health care transition; health care disparities; disparity; adult health; elderly health; aging adults; health equity; public health; health parity; minority populations

Submitted August 3, 2016; submitted, revised, March 16, 2017; accepted April 26, 2017.

Funding support: J.A.P. was supported by the Ohio State University College of Medicine Roessler Research Scholarship. S.M.H. was supported by the Centers for Disease Control and Prevention Cooperative Agreement No. 2U59DD000931-04 and the Maternal and Child Health Bureau of Health Resources and Services Administration under award no. T73MC24481-02. E.A.Y. was supported by the Centers for Disease Control and Prevention Cooperative Agreement Number 2U59DD000931-04.
Disclaimer: The contents of this article are solely the responsibility of the authors and do not necessarily represent the official views of the Centers for Disease Control and Prevention.

Acknowledgments: Katherine Randle and Rebecca McAdams, MA, $\mathrm{MPH}$ are acknowledged for their contributions to this article.

Supplementary materials: Available at http://www.AnnFamMed. org/content/15/5/471/suppl/DC1/.

\section{References}

1. Developmental Disabilities Assistance and Bill of Rights Act of 2000, Public Law No. 106-402, §102 Stat 1683-1684.

2. Wang $G$, McGrath BB, Watts $C$. Health care transitions among youth with disabilities or special health care needs: an ecological approach. J Pediatr Nurs. 2010;25(6):505-550.

3. Dejong G, Palsbo SE, Beatty PW, Jones GC, Knoll T, Neri MT. The organization and financing of health services for persons with disabilities. Milbank Q. 2002;80(2):261-301.

4. Havercamp SM, Scandlin D, Roth M. Health disparities among adults with developmental disabilities, adults with other disabilities, and adults not reporting disability in North Carolina. Public Health Rep. 2004;119(4):418-426.

5. Ohio Colleges of Medicine Government Resource Center and RTI International. 2015 Ohio Medicaid Assessment Survey: methodology report. http://grc.osu.edu/sites/default/files/inline-files/120150 MASMethReptFinal121115psg.pdf. Published Dec 8, 2015. Accessed Jul 12, 2016.

6. Cooley WC, Sagerman PJ; American Academy of Pediatrics; American Academy of Family Physicians; American College of Physicians; Transitions Clinical Report Authoring Group. Supporting the health care transition from adolescence to adulthood in the medical home. Pediatrics. 2011;128(1):182-200.

7. Steward D, Freeman M, Law M. Transition to adulthood for youth with disabilities: evidence from the literature. In: Stone JH, Blouin M, eds. International Encyclopedia of Rehabilitation. http://cirrie.buffalo.edu/encyclopedia/en/article/110/. Published 2010. Accessed Jul 12,2016

8. Ohio Colleges of Medicine Government Resource Center and RTI International. 2015 Ohio Medicaid Assessment Survey: adult and child CATI instrument specifications. http://www.shadac.org/sites/ default/files/OH_2015_HH_instrument.pdf. Published Dec 8, 2015. Accessed Jul 14, 2016.

9. Holder M, Waldman HB, Hood H. Preparing health professionals to provide care to individuals with disabilities. Int J Oral Sci. 2009;1(2):66-71.

10. Symons AB, McGuigan D, Akl EA. A curriculum to teach medical students to care for people with disabilities: development and initial implementation. BMC Med Educ. 2009;9(78):78. 